\title{
Asciminib mitigates DNA damage stress signalling induced by cyclophosphamide in the ovary
}

\author{
Luca Mattiello ${ }^{1}$, Giulia Pucci ${ }^{1}$, Francesco Marchetti ${ }^{1}$, Marc Diederich ${ }^{2}$ and Stefania Gonfloni ${ }^{1, *}$ \\ 1 Department of Biology, University of Rome Tor Vergata, via della Ricerca Scientifica, 00133 Rome, Italy; \\ luca.mattiello@alice.it (L.M.); giulia.pucci94@gmail.com (G.P.); francescomarchetti@icloud.com (F.M.) \\ 2 College of Pharmacy, Seoul National University, 1 Gwanak-ro, Gwanak-gu, Seoul 08826, Republic of Korea, \\ marcdiederich@snu.ac.kr \\ * Correspondence: stefania.gonfloni@uniroma2.it
}

Abstract: Cancer treatments often have adverse effects on the quality of life for young women. One of the most relevant negative impacts is the loss of fertility. Cyclophosphamide is one of the most detrimental chemotherapeutic drugs for the ovary. Cyclophosphamide may induce the destruction of dormant follicles while promoting follicle activation and growth. Herein, we demonstrate the in vivo protective effect of the allosteric Bcr-Abl tyrosine kinase inhibitor Asciminib on signalling pathways activated by cyclophosphamide in mouse ovaries. Besides, we provide evidence that Asciminib did not interfere with the cytotoxic effect of cyclophosphamide in MCF7 breast cancer cells. Our data indicate that concomitant administration of Asciminib mitigates the cyclophosphamide-induced ovarian reserve loss without preventing the anticancer potential of cyclophosphamide. Altogether these observations are relevant for the development of effective ferto-protective adjuvants to preserve the ovarian reserve from the damaging effect of cancer therapies.

Keywords: ovarian reserve, cyclophosphamide, DNA damage response, drug repurposing, allosteric tyrosine kinase inhibitors, Asciminib

\section{Introduction}

Chemotherapy, radiation, or combinations of them are commonly used in cancer therapy. Ovarian failure and infertility are well-known side effects of such therapies [1-3]. Cyclophosphamide (Cy) is an alkylating chemotherapeutic drug routinely used against solid and hematological malignancies [4]. Recent studies highlighted the primary target of $\mathrm{Cy}$ in vivo in murine ovaries [5-8]. Cyclophosphamide exposure induced death of growing granulosa cells and the concomitant activation of the DNA Damage Response (DDR) and AKT-FOXO3a signaling axis in the nucleus of reserve oocytes [6]. These observations supported the hypothesis that damaging stress pathways are activated in a concomitant manner both in somatic and germ cells following chemotherapy. However, the identification of sentinel molecules directly involved in communicating stress signalling remains a daunting task, as the physiological changes in the ovary reflect both direct or indirect effects of genotoxic assaults. Despite this, understanding the molecular mechanisms underlying ovarian reserve loss induced by cancer therapies remains essential for developing a more effective treatment to preserve the fertility of female patients.

2. Results 
In this study, we evaluated the effects of $\mathrm{Cy}$ (or of an active metabolite 4-hydroperoxycyclophosphamide, 4-OH-Cy) alone and in combination with Asciminib, either in vivo in mice or in vitro in a model cell line for breast cancer (MCF7). First, we validated the re-localization of c$\mathrm{Abl}$ after treatment with Asciminib as previously described for the allosteric inhibitor GNF2 [9]. We monitored the c-Abl localization in a transgenic MEF line (Mouse Embryonic Fibroblast, lacking the expression of $\mathrm{c}-\mathrm{Abl}$ ) following transfection with a c-Abl expression vector. We evaluated the c-Abl localization by immunofluorescence (IF) assay in transfected MEF cells, treated with different c-Abl inhibitors. We tested either allosteric ligands such as GNF2, Asciminib, and one ATP-binding competitive inhibitor, Imatinib. IF assays clearly showed that Asciminib, as well as GNF2, induced a re-localization of c-Abl in the perinuclear zone as indicated by the yellow arrows (Figure 1). On the contrary, treatment with Imatinib did not cause any enrichment of c-Abl kinase in the perinuclear zone.

A

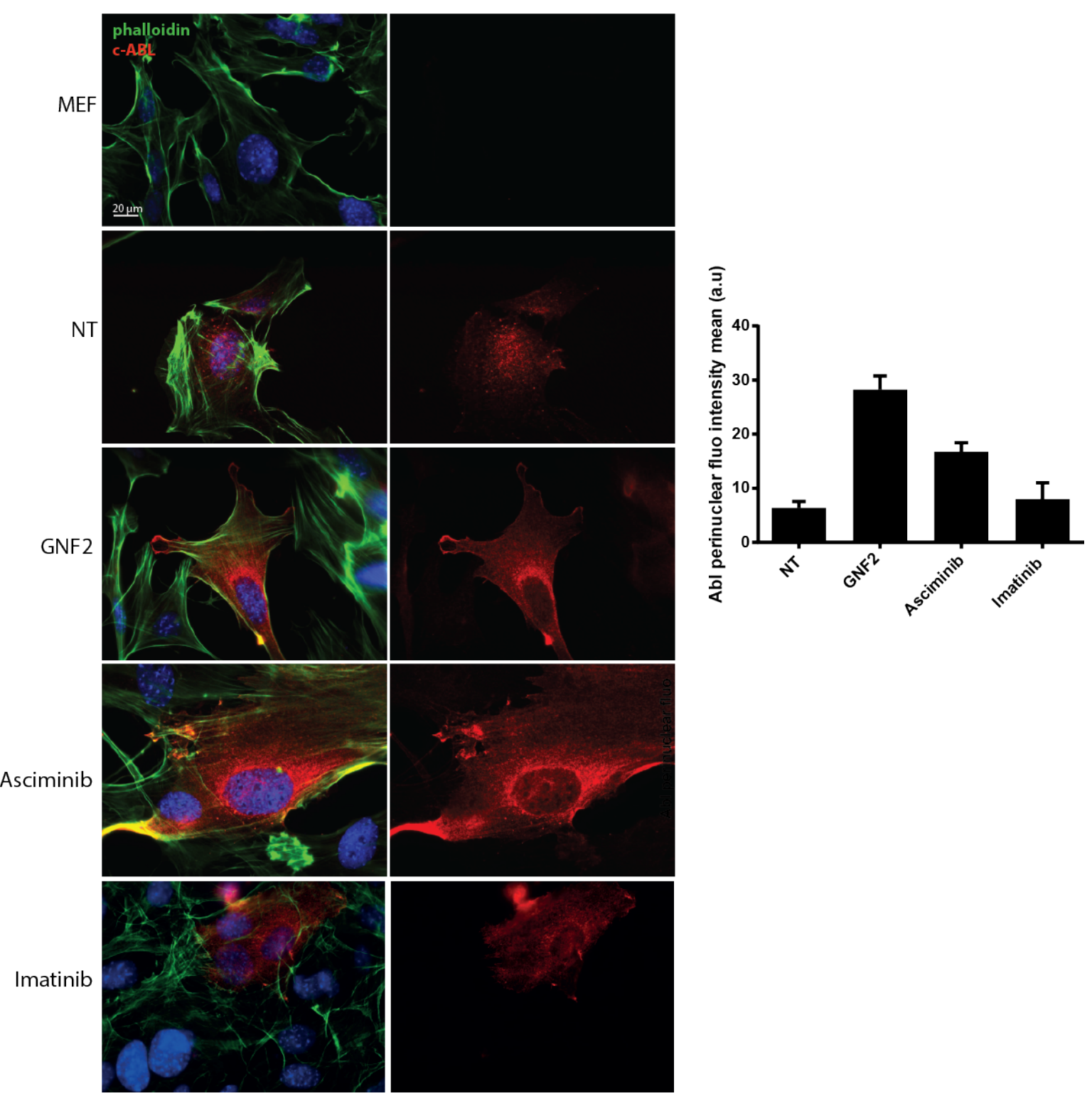


c-Abl. The yellow arrows indicated the re-localization of c-Abl tyrosine kinase in the perinuclear zone induced by Asciminib or GNF2 exposure. Bar column represents mean \pm s.e.m. Scale bar 20 $\mu \mathrm{m}$.

Asciminib modulated the DDR and the follicle activation induced by Cy exposure in vivo.

Next, we injected P7 mice with Cy alone $(100 \mathrm{mg} / \mathrm{kg})$ or in combination with increasing concentrations of Asciminib (0.1, 0.2, and $0.5 \mathrm{mg} / \mathrm{kg}$, respectively). At different time points, the ovaries were dissected, lysed, and analyzed by IF or western blot (W.B.) assays. Co-treatment with Asciminib resulted in partial inhibition of TAp63 phosphorylation (commonly observed as a shift of TAp63 protein by W.B. assay). In Figure 2A, we observed a partial prevention of TAp63 shift (see black arrows) 18 hours after co-injection of Cy and Asciminib. We observed the phosphorylation of histone H2AX at Ser139 $(\gamma \mathrm{H} 2 \mathrm{AX})$, an early marker of DDR in the ovarian lysates. Of note, co-treatment with Asciminib affected the $\gamma \mathrm{H} 2 \mathrm{AX}$ phosphorylation as assessed by W.B. assay (Supplementary Figure 1).

To assess whether Asciminib may affect DDR activation in primordial/primary oocytes, we monitored the phosphorylation of DDR sentinel proteins by IF assays performed on ovarian sections. We found that Asciminib attenuated DNA stress signalling induced by $\mathrm{Cy}$ in the ovarian reserve. Co-treated ovaries showed reduced staining for phospho-DNA-PK, $\gamma \mathrm{H} 2 \mathrm{AX}$ and cleaved PARP in the nucleus of reserve oocytes (Figure 2 panels B, C, D). Furthermore, co-treatment of reserve oocytes with Asciminib and Cy prevented nuclear AKT phosphorylation (Figure $2 \mathrm{E}$ ). Taken together, these data demonstrated that Asciminib modulated signalling pathways activated by $\mathrm{Cy}$ in the ovary. 
A

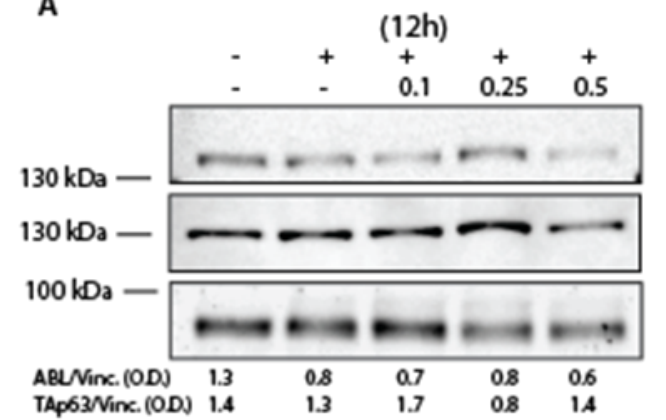

Cy

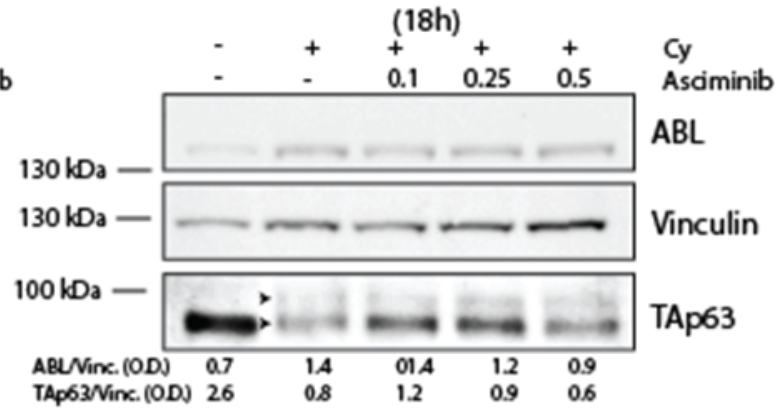

ABL

Vinculin

TAp63

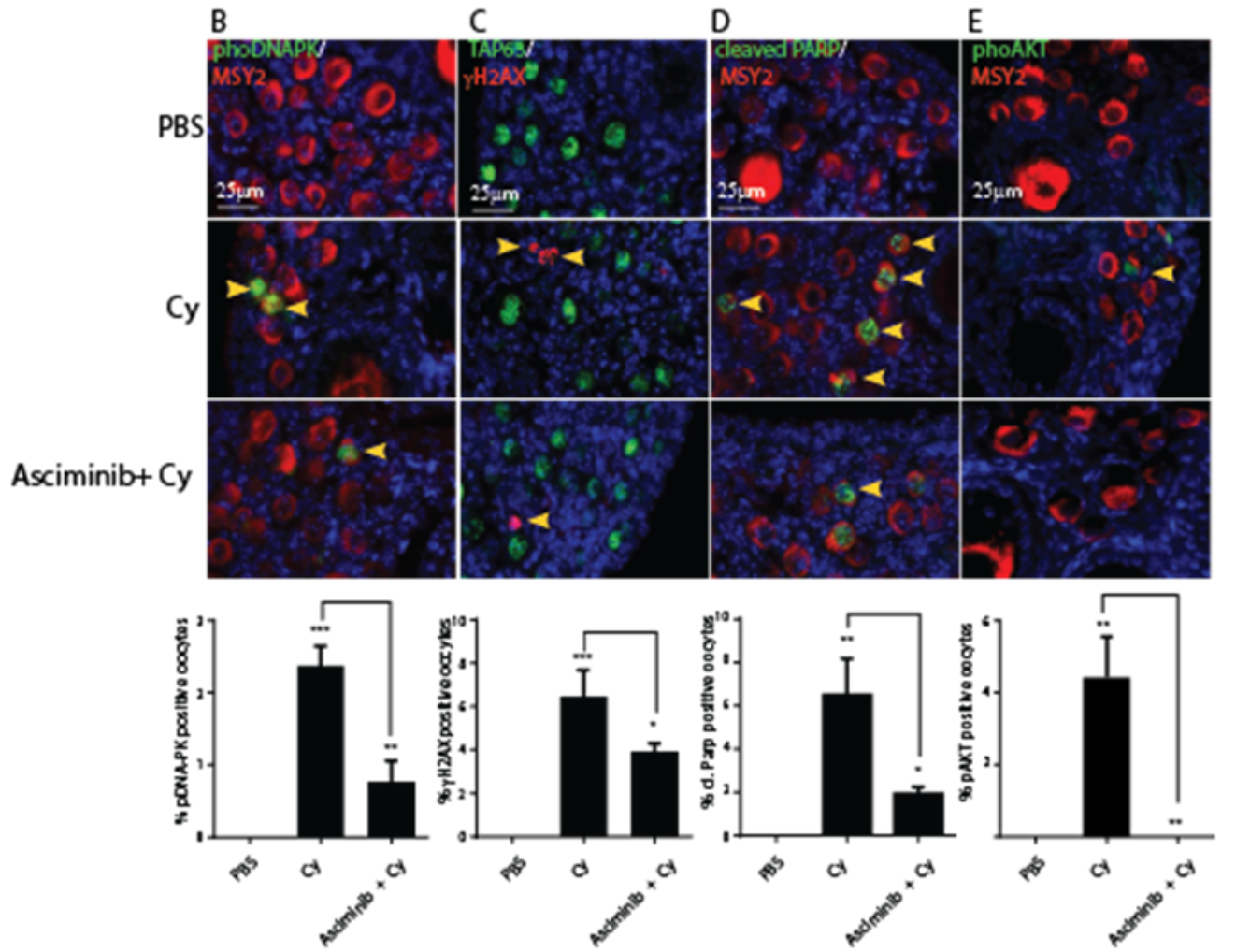

Figure 2. Asciminib mitigated both DDR and apoptosis induced by $\mathrm{Cy}$ in the ovarian reserve. (A) W.B. analysis of ovarian lysates from female pups injected with Cy $100 \mathrm{mg} / \mathrm{kg}$ alone or in combination with various doses of Asciminib and collected at different time points. P7 mice were injected with vehicle (PBS) or Cy $(100 \mathrm{mg} / \mathrm{kg}$ ) alone or in tandem with Asciminib $(0.25 \mathrm{mg} / \mathrm{kg})$ and sacrificed within $16 \mathrm{~h}$ from injection. Numerical values, under the blots, represent band densitometries normalized on the housekeeping gene. (B) DNA-PK activation was evaluated by IF assay using phospho-specific antibodies (green), while Msy2 (red) was used as a cytoplasmic marker for germ cells. (C) $\gamma \mathrm{H} 2 \mathrm{AX}$ phosphorylation was evaluated by IF assay using phosphospecific antibodies (red), while p63 (green) was used as a nuclear marker for germ cells (D) Follicle reserve apoptosis was assessed by IF assay with two specific antibodies against cleaved PARP (green) and Msy2 (red). (E) The follicular activation was assessed by IF assay with specific phospho-antibodies for AKT (T308) (green) and Msy2 (red). Quantification was performed by counting several $(6<x<8)$ middle ovarian sections derived from three distinct ovaries. Scale Bar magnification, $25 \mu \mathrm{m}$. Bar column represents mean \pm s.d.; statistical significance was determined 
bioRxiv preprint doi: https://doi.org/10.1101/2020.12.30.424825; this version posted December 30, 2020. The copyright holder for this preprint (which was not certified by peer review) is the author/funder. All rights reserved. No reuse allowed without permission.
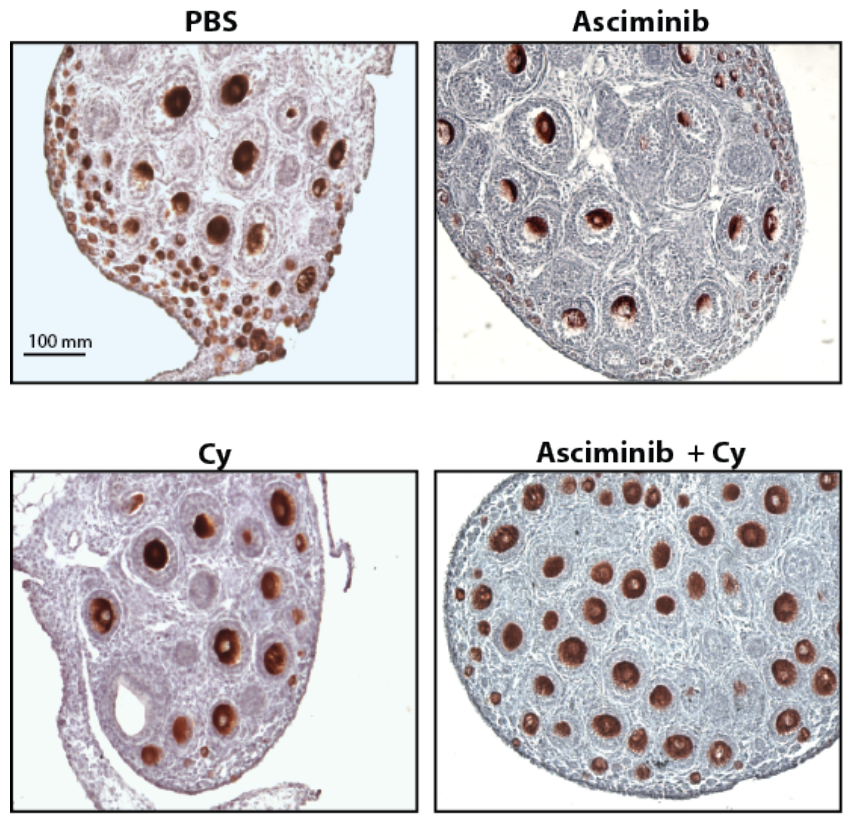
PBS-treated group). a toxic effect per se. using one-way analysis of variance (ANOVA) $\left({ }^{*} \mathrm{P}<0.05 ;{ }^{* *} \mathrm{P}<0.01 ;{ }^{* *} \mathrm{P}<0.001\right.$ compared with

\section{Asciminib protected the ovarian reserve following Cy treatment.}

Next, we injected female P6 pups with Cy $(100 \mathrm{mg} / \mathrm{kg})$ alone or in combination with Asciminib $(0.25 \mathrm{mg} / \mathrm{kg})$. Three days after injection, we collected ovaries to perform immunohistochemistry (IHC) assays with an antibody against cytoplasmic germ cell antigen (Msy2) (red) (Figure 3). We counted the follicle reserve from mid-ovary sections of different ovaries. IHC assays of ovarian sections showed a massive depletion of primordial and primary follicles in Cy-treated mice, whereas a $\mathrm{Cy}+\mathrm{Asciminib}$ co-treatment significantly rescued reserve follicles. Follicle protection was dependent on the concentration of Asciminib, as shown in Supplementary Figure 2A, higher dosage of Asciminib (1 mg/kg) did not prevent follicle death induced by $\mathrm{Cy}$ and seemed to have

Figure 3. Asciminib protected the ovarian reserve from $\mathrm{Cy}$ treatment. Ovaries of each $100 \mathrm{mg} / \mathrm{kg}$ alone or in tandem with Asciminib $0.25 \mathrm{mg} / \mathrm{kg}$ ) and analyzed by IHC assay with Msy2 antibody. Several ovaries from independent experiments were analyzed. Each dot in the box plot represents the average primordial+primary follicles numbers per section of each gonad collected. Statistical significance was determined using one-way analysis of variance (ANOVA) $\left({ }^{* *} \mathrm{P}<0.01\right.$ compared to Cy $100 \mathrm{mg} / \mathrm{kg}$ ). Scale Bar, $100 \mu \mathrm{m}$.

\section{Asciminib did not prevent the DNA damage induced by Cy in MCF7}

A clinically-used ferto-protective drug should not interfere with the therapeutic effect of DNAdamaging chemotherapies. We validated this assumption by assessing the effect of Asciminib on 4-hydroperoxy-cyclophosphamide (4-OH-Cy)-treated MCF7 breast tumor cells. Our results showed that the co-treatment with Asciminib did not affect 4-OH-Cy-induced phosphorylation of DDR marker proteins like ATM, $\gamma \mathrm{H} 2 \mathrm{AX}$ or p53 (Figure 4A). Besides, single-cell gel 
electrophoresis (Comet) assays showed that Asciminib did not interfere with the DNA-damaging effect of 4-OH-Cy (Figure 4B). Lastly, the co-administration of Asciminib did not affect the cytotoxic effect of 4-OH-Cy (Figure 4C). Altogether these data support the potential use of

A

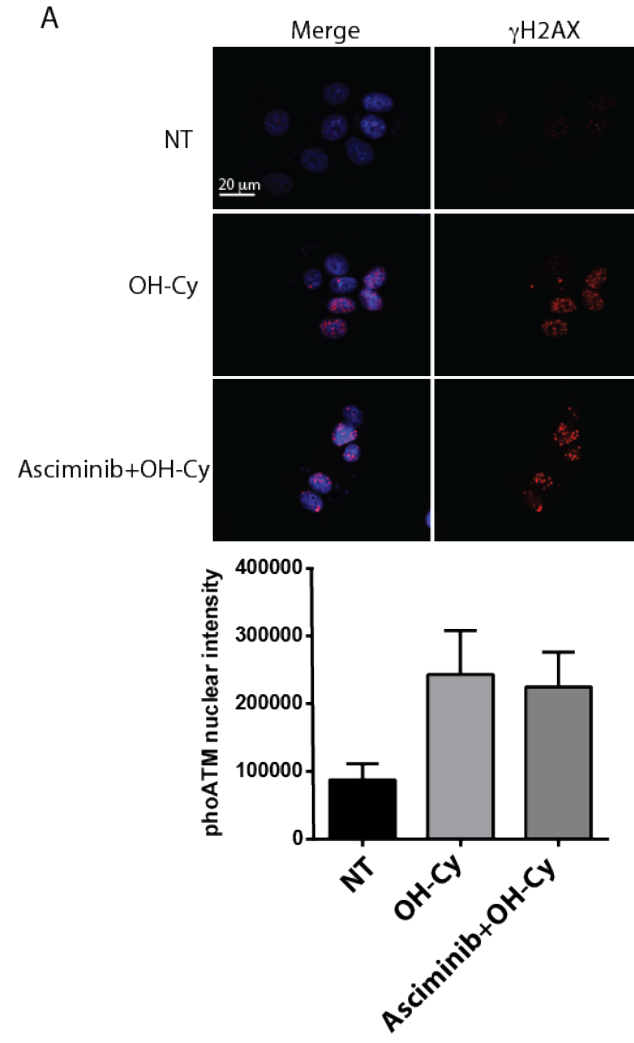

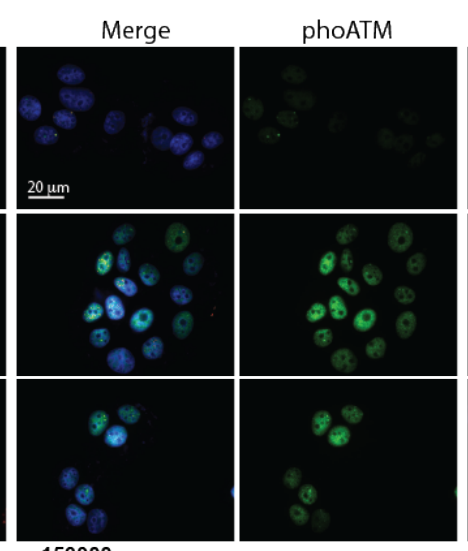

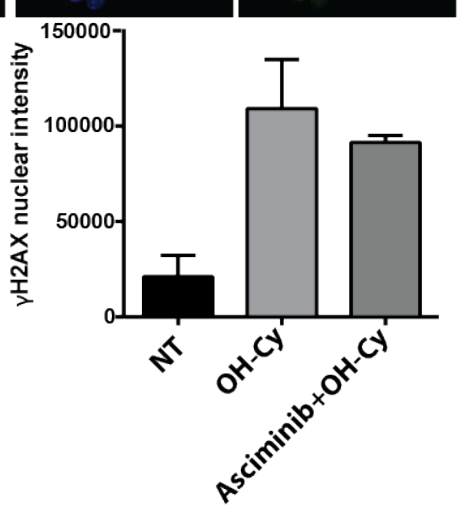

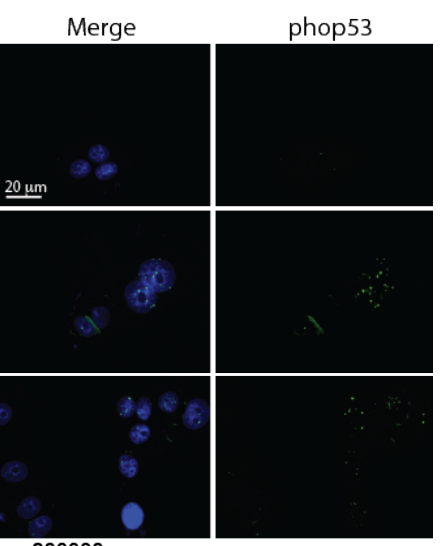

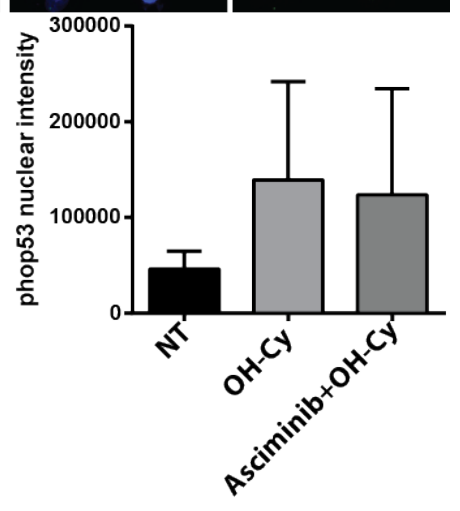

B

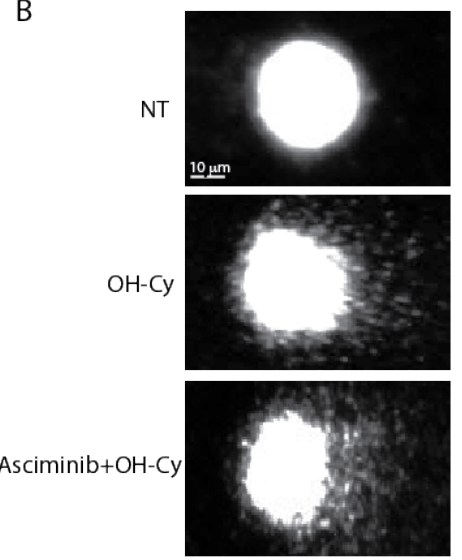

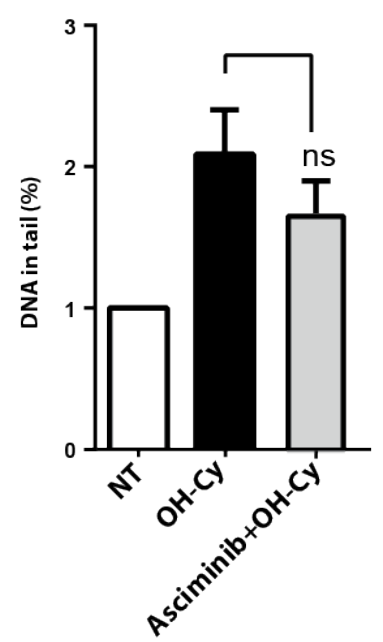

C

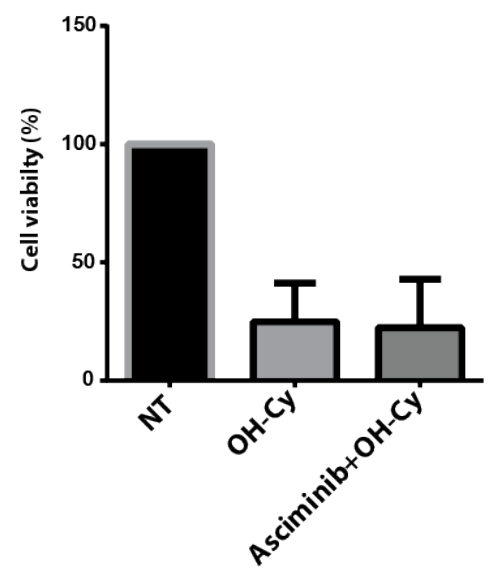

Figure 4. Asciminib did not prevent the cytotoxicity of 4-OH-Cy in MCF7. MCF7 cells were treated with Asciminib $(0.5 \mu \mathrm{m})$. After 1 hour, 4-OH-Cy was added in the medium (4-OH-Cy 10 $\mu \mathrm{M}$ for comet and IF assay, 4-OH-Cy $50 \mu \mathrm{M}$ for MTS assay. (A) DDR signalling was evaluated by IF after 4 hours of 4-OH-Cy treatment using specific phospho-antibodies for sentinel proteins. Bar columns represent mean \pm s.e.m.; nuclear fluorescence intensity was evaluated by ImageJ 
software. Scale bar $20 \mu \mathrm{m}$. (B) DNA fragmentation was assessed by comet assay following 4 hours of 4-OH-Cy treatment; DNA percentage in tail quantification was evaluated by Comet Score software. Bar columns represent mean \pm s.e.m.; Scale bar $10 \mu \mathrm{m}$. (C) Drug toxicity was measured by MTS assay after 48 hours of $4-\mathrm{OH}-\mathrm{Cy}$ treatment. Bar columns represent mean \pm s.e.m.

\section{Discussion}

In young women, chemotherapy regimens increase the risk of premature ovarian failure (POF) and infertility. Genotoxic agents have two different effects on ovarian function: the first one is immediate, induces amenorrhea and the loss of growing follicles; the second one has a long-term impact by inducing a loss of the ovarian reserve. While the first effect is reversible, the loss of the follicle reserve is permanent and leads to infertility. As such, fertility preservation is an urgent issue for cancer survivors. Pharmacological agents can prevent follicle loss at the time of treatment while providing several advantages over fertility conservation techniques. These small molecules could act as ferto-protective drugs [10] by counteracting the effects of chemotherapy on the ovarian reserve. Ferto-protective drugs may be suitable for patients of all ages while avoiding the use of invasive hormonal and surgical procedures. Such ferto-adjuvants should not interfere with chemotherapeutic treatments while preventing endocrine side effects of premature ovarian failure and infertility [11]. Primordial follicles maintain their genome integrity for several decades in human without relying on classical DNA quality check controls of somatic cells during the cell cycle [12]. A better understanding of the signalling pathways activated in the follicle reserve (formed by a single oocyte surrounded by few granulosa cells) following chemotherapy is fundamental for the development of effective ferto-protective drugs. Recent findings from Rinaldi et al suggested that the oocytes reaching a threshold level of unrepaired DSBs after irradiation can be eliminated by a signalling pathway that required both p53 and TAp63 $\alpha$ transcription factors [13]. Compelling evidence suggested a role of c-Abl in the oocyte degeneration induced by chemotherapy [14-16]. In mice, pharmacological inhibition of c-Abl counteracted the cytotoxic effect of cisplatin [14, 16, 17] and cyclophosphamide [6]. In this study, we tested a novel selective allosteric compound targeting c-Abl, Asciminib, against the damaging effects of cyclophosphamide. Allosteric inhibitors bind a deep pocket in the large lobe of the cAbl catalytic domain, which is very far from the ATP binding site. Of note, small allosteric ligands induced conformational changes even in the ATP binding pocket of the kinase, preventing its active conformation [18]. Asciminib is a novel and more selective inhibitor compared to GNF2 [19]. Also, Asciminib is already used in phase III assays (NCT03106779) against resistant forms of Chronic Myeloid Leukemia (CML) [20]. Here, we showed that the co-administration of Asciminib had a protective effect on the ovarian reserve, as assessed by immunohistochemistry (IHC) performed three days after Cy injection. Besides, we found that Asciminib did not counteract the genotoxic effect exerted by an active $\mathrm{Cy}$ active metabolite (4-OH-Cy) on breast cancer cells. Together these data support the potential use of Asciminib as a ferto-adjuvant during chemotherapeutic regimens.

\section{Materials and Methods}

Animals and injection

All procedures involving mice and care have been conducted at the Interdepartmental Service Centre-Station for Animal Technology (STA), University of Rome "Tor Vergata", in accordance with the ethical standards, according to the Declaration of Helsinki, in compliance with our institutional animal care guidelines and following national and international directives (Italian Legislative Decree 26/2014, Directive 2010/62/E.U. of the European Parliament and of the Council). The ovaries were collected from CD-1 mice (Charles River) of 6 to 8 days old. Newborn 

weight). Mice were pre-treated with Asciminib (0,1-0,5 mg per kg of body weight). Cy (BAXTER) was fresh prepared at $40 \mathrm{mg} / \mathrm{ml}$ in PBS. We dissolved Asciminib in DMSO.

We prepared sections from ovaries fixed in MetaCarnoy solution, embedded in paraffin and cut in slices of $7 \mu \mathrm{m}$ of thickness. Sections were dewaxed, re-hydrated, and microwaved. Slices were then permeabilised with PBS triton 0,2 \% and incubated with MSY-2 antibody (Santa Cruz). The staining was performed with immunocruz staining system for anti-goat antibody (Santa Cruz, sc-2023) and 3-aminoethyl-9-ethylcarbazole as substrate (AEC, Sigma). Sections were counterstained with hematoxylin and cover-slipped with Aquatex. Quantification of primordial and primary or secondary follicles was derived from histological analysis, counting Msy2positive germ cells of mid-ovary sections. For each ovary (derived from P9 mice P9 pups), several central slices $(10<\mathrm{n}<15)$ are included in the counting, except for smaller peripheral slices $(12-14$ on average per each ovary). Quantification of primordial/primary follicle reserve is expressed as mean of immature follicles (primordial plus primary follicles) per single ovary. Average values for each ovary are represented as discrete points on a scatter plot. Mean value \pm S.D. are shown in the scatter plot. The analysis of variance is evaluated with one-way ANOVA, with Turkey multiple comparison Test using PRISM 6 (Graph Pad software) $\left({ }^{*} \mathrm{P}<0.05 ;{ }^{* *} \mathrm{P}<0.01\right.$; ${ }^{* *} \mathrm{P}<0.001$ ) or by unpaired Student's $t$ test where indicated.

We prepared sections from ovaries sections fixed in MetaCarnoy solution, embedded in paraffin and cut in slice of $7 \mu \mathrm{m}$ of thickness. Sections were dewaxed re-hydrated and microwaved in sodium citrate $10 \mathrm{mM} \mathrm{pH} 6$, to expose the antigens. Unspecific-binding sites were blocked by incubating sections for 2 hours in a blocking solution (PBS plus 1\% glycine 5\% FBS and 5\% NGS (normal goat serum). Ovaries sections were then incubated overnight with antibodies against MSY-2, p63, $\gamma \mathrm{H} 2 \mathrm{AX}$, p-DNA-PK, pAKT and cleaved PARP. After washing in PBS triton 0,05\%, tissue sections were incubated with Alexa 555-goat anti-mouse (life technologies) and Alexa 488goat anti rabbit (Invitrogen). Nuclei were stained with $1 \mu \mathrm{g} / \mathrm{mL}$ Hoechst 33342 dyes for cells (Thermo Fischer Scientific) in PBS 1X.

P7 dry ice-frozen ovaries were homogenized with a mini-pestle in ice-cold lysis buffer $(50 \mathrm{mM}$ Tris- $\mathrm{HCl} \mathrm{pH}$ 7.5, $150 \mathrm{mM} \mathrm{NaCl}, 0.5 \% \mathrm{NP}-40,5 \mathrm{mM}$ EDTA, 0.5\% sodium deoxycholate, $1 \mathrm{mM}$ phenylmethylsulfonyl fluoride, $1 \mathrm{mM}$ sodium o-vanadate, $10 \mu \mathrm{g}$ ml-1 Tosyl phenylalanyl chloromethyl ketone (TPCK), $10 \mu \mathrm{g}$ ml-1, Tosyl-L-lysyl-chloromethane hydrochloride (TLCK) supplemented with protease inhibitors, (all purchased from SIGMA). Equal amounts of protein extract (equivalent of one up to three ovaries) was loaded onto $6 \%, 8 \%$ or $12 \%$ SDS-PAGE gel and transferred to a nitrocellulose membrane (Amersham Bioscience). Immunoblot densitometry were performed using ImageJ software. 
MEF Abl-/- cells were kindly gifted by the Koleske lab (Yale, USA). MCF7 cells were kindly provided by the Barilà group (IRCCS-Fondazione Santa Lucia, Rome, Italy). Cells were grown in DMEM medium (GIBCO) supplemented with 15\% FBS (Mef Abl-/-) or 10\% FBS (for MCF7) (Lonza) and $100 \mathrm{U} / \mathrm{ml}$ penicillin/streptomycin (Lonza) in a humidified atmosphere containing 5\% $\mathrm{CO}_{2}$ at $37^{\circ} \mathrm{C}$.

Cells transfection and Immunofluorescence assay

MEF Abl-/- were grown in a 6-wells plate on cover slips and transfected with a plasmid encoding wild-type c-Abl $1 \mathrm{~b}$ isoform for 16 hours (at low passages), by using lipofectamine 2000 DNA Transfection Reagent (Invitrogen) according to the manufacturer's instruction. During this time the cells were also treated with small-molecule c-Abl compounds alone (DPH, GNF-2, Asciminib) for 4 hours and then analyzed by Immunofluorescence.

MCF-7 cultured in a 6-wells plate on cover slips were incubated for 60 minutes with allosteric cAbl inhibitors, Asciminib $(0.5 \mu \mathrm{M})$ or GNF-2 $(10 \mu \mathrm{M})$, treated with 4hydroperoxycyclophosphamide (4-OH-Cy) (sc-206885) for 4 hours and analyzed by Immunofluorescence.

Cells were grown on cover slips, then washed in PBS and fixed with $4 \%$ paraformaldehyde for $10 \mathrm{~min}$ at room temperature. Cells were incubated with a solution containing Triton X-100 (0.5\%), blocked for 2 hours with a blocking solution (PBS, Triton X-100 0.1\%, BSA 5\%), and then incubated with primary antibodies against p-ATM, $\gamma \mathrm{H} 2 \mathrm{AX}$ and p-p53 for 60 minutes. After washing in PBS/Triton $0,1 \%$, cells were incubated with Alexa 555-goat anti-mouse (Life technologies) and Alexa 488-goat anti rabbit (Invitrogen) for 30 minutes. Nuclei were stained with $1 \mu \mathrm{g} / \mathrm{ml}$ 4,6-diamidino-2-phenylindole dihydrochloride (Thermo Fischer Scientific). Fluorescence images were obtained by Leica DMR Fluorescence Microscope (Leica Microsystems, Germany). Perinuclear fluorescence analysis was performed using FIJI software. In particular, the regions of interest (ROI) were identified using DAPI signal to spot the edge of nuclear region. From this edge, a 30 pixels-wide area was pointed out using the fix ellipse command and then used as ROI to measure fluorescence intensity mean.

Breast cancer cells (MCF7) cultured in $60 \mathrm{~mm}$ dishes were incubated for 60 minutes with allosteric inhibitors, Asciminib $(0.5 \mu \mathrm{M})$ or GNF-2 $(10 \mu \mathrm{M})$, treated with 4-hydroperoxycyclophosphamide (OH-Cy) for 4 hours. All following steps were conducted under dim light to prevent the occurrence of additional DNA damage. The cells were washed with PBS twice and $20 \mu$ l of each cellular lysate were mixed with $730 \mu \mathrm{l}$ of $0,5 \%$ low melting agarose solution. One tenth of this volume was dropped on slides coated with $1 \%$ normal melting agarose. The slides were covered with coverslips and placed on ice for $5 \mathrm{~min}$. After gel solidification coverslips were gently removed. The slides were placed into Schifferdecker type glass cuvette, filled with lysis solution (10 mmol/L Tris- $\mathrm{HCl}, 2.5 \mathrm{~mol} / \mathrm{L} \mathrm{NaCl}, 100 \mathrm{mmol} / \mathrm{L}$ EDTA, 1\% Triton-X 100, 10\% DMSO, pH 10, $4^{\circ} \mathrm{C}$ ) and incubated at $4{ }^{\circ} \mathrm{C}$ overnight. After lysis step, the slides were washed with deionized water and placed into electrophoresis chamber (Apelex) filled with $2.2 \mathrm{~L}$ of alkaline 
electrophoretic solution ( $300 \mathrm{mmol} / \mathrm{L} \mathrm{NaOH}, 1 \mathrm{mmol} / \mathrm{L}$ EDTA, $4^{\circ} \mathrm{C}, \mathrm{pH}>13$,) for $30 \mathrm{~min}$. Electrophoresis was performed in the same solution for $20 \mathrm{~min}$ at electric field strength of 0,7 $\mathrm{V} / \mathrm{cm}$. The applied voltage was $11 \mathrm{~V}$ and the current was $280 \mathrm{~mA}$. After electrophoresis, the slides

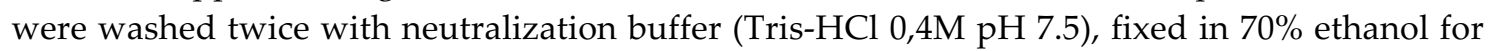
10 minutes, dried at room temperature and stored until staining. Immediately prior to microscopic analysis, the slides were stained with GelRed (SIGMA) in the dark. The images of comets were analyzed using Comet Score software and approximately 300 cells per slide were counted. DNA fragmentation was evaluated by the percentage of DNA in the tail of comet (\% DNA tail).

MCF7 cells were seeded into 96-wells plates at a density of 10000 cells per well $(200 \mu \mathrm{l})$ and treated with the following: vehicle control (DMSO), $\mathrm{OH}-\mathrm{Cy}$ alone or in combination with Asciminib $(0.5 \mu \mathrm{M})$. The cells were treated for $24 \mathrm{~h}$. For the MTS assay, the CellTiter $96 \AA$ AQueous One Solution Cell Proliferation Assay kit (Promega, USA) was used following the manufacturer's instruction. The absorbance of cells for each condition was detected at $490 \mathrm{~nm}$ with a microplate reader (Sunrise Tecan microplate reader).

Antibodies for Msy-2 (sc-21316) and p-AKT (T308) (sc-16646-R) were purchased from Santa Cruz; antibody for p-H2AX ( $\gamma \mathrm{H} 2 \mathrm{AX})(05-636), \mathrm{H} 2 \mathrm{AX}$ (07-627) and were purchased from Millipore; antibody for p-DNA-PK (S2056) (SAB4504169) was purchased from SIGMA, antibody for p-ATM (S1981) (200-301-500) was purchased from Rockland, polyclonal antibody for p63 was a homemade rabbit serum; antibody for p-P53 (S15) (9284) and cleaved PARP (9544) were purchased from Cell Signalling Technology. Western Blot secondary antibodies were purchased from Jackson Immunoresearch. All the antibodies were diluted in a blocking solution containing 5\% BSA in PBS tween 0,05\% for Western Blotting analysis and in a blocking solution containing $1 \%$ glycine, 5\% FBS and 5\% NGS for immunofluorescence assay.

Author Contributions: L.M. performed the majority of the experiments with the help of F.M. G.P. performed MTS and IF assays in MCF7 for phoATM, phop53, $\gamma$ H2AX. M.D. provided reagents and critical reading of the paper. S.G. designed, directed the study and wrote the paper.

Funding: This work was supported by grants from AIRC (IG11344) to S.G.

Acknowledgments: We thank Luisa Castagnoli for her valuable support. We are indebted to Stefano Cannata e Sergio Bernardini for technical advice. M.D. is supported by the National Research Foundation (NRF) (grant number 019R1A2C1009231), by a grant from the MEST of Korea for Tumour Microenvironment Global Core Research Center (GCRC) (grant number NRF-2011-0030001), by the Creative-Pioneering Researchers Program through Seoul National University (Funding number: 370C20160062), by the Brain Korea 21 (BK21) PLUS programme, by the 'Recherche Cancer et Sang' foundation, by the 'Recherches Scientifiques Luxembourg' association, by the 'Een Häerz fir kriibskrank Kanner' association, by the Action LIONS 'Vaincre le Cancer' association and by Télévie Luxembourg. 
303 1. Oktem, O.; Oktay, K., Quantitative assessment of the impact of chemotherapy on ovarian follicle 304 reserve and stromal function. Cancer 2007, 110, (10), 2222-9.

305 2. Meirow, D.; Biederman, H.; Anderson, R. A.; Wallace, W. H., Toxicity of chemotherapy and radiation on female reproduction. Clin Obstet Gynecol 2010, 53, (4), 727-39.

307 3. Johnson, E. K.; Finlayson, C.; Rowell, E. E.; Gosiengfiao, Y.; Pavone, M. E.; Lockart, B.; Orwig, K. E.; Brannigan, R. E.; Woodruff, T. K., Fertility Preservation for Pediatric Patients: Current State and Future Possibilities. J Urol 2017, 198, (1), 186-194.

4. Sonmezer, M.; Oktay, K., Fertility preservation in female patients. Hum Reprod Update 2004, 10, (3), 25166.

5. Kalich-Philosoph, L.; Roness, H.; Carmely, A.; Fishel-Bartal, M.; Ligumsky, H.; Paglin, S.; Wolf, I.; Kanety, H.; Sredni, B.; Meirow, D., Cyclophosphamide Triggers Follicle Activation and "Burnout"; AS101 Prevents Follicle Loss and Preserves Fertility. Sci Transl Med 2013, 5, (185), 185ra62.

Bellusci, G.; Mattiello, L.; Iannizzotto, V.; Ciccone, S.; Maiani, E.; Villani, V.; Diederich, M.; Gonfloni, S., and prolongs fertility. Cell Death Dis 2019, 10, (10), 726. from cyclophosphamide. J Endocrinol 2019, 240, (2), 243-256. ovarian follicles following phosphoramide mustard exposures of cultured rodent ovaries in vitro. Toxicol Appl Pharmacol 2011, 253, (2), 94-102.

326 10. Woodruff, T. K., Preserving fertility during cancer treatment. Nat Med 2009, 15, (10), 1124-5.

327 11. Roness, H.; Kashi, O.; Meirow, D., Prevention of chemotherapy-induced ovarian damage. Fertil Steril 2016, 105, (1), 20-9.

329 12. Gonfloni, S., Modulating c-Abl nuclear activity as a strategy to preserve female fertility. Cell cycle 2010, $3309,(2), 217-8$.

331 13. Rinaldi, V. D.; Bloom, J. C.; Schimenti, J. C., Oocyte Elimination Through DNA Damage Signalling from CHK1/CHK2 to p53 and p63. Genetics 2020, 215, (2), 373-378. 
primordial follicle oocyte killing and loss of fertility are not prevented by imatinib. Nat Med 2012, 18, (8), 1172-4.

15. Vandormael-Pournin, S.; Guigon, C. J.; Ishaq, M.; Coudouel, N.; Ave, P.; Huerre, M.; Magre, S.; CohenTannoudji, J.; Cohen-Tannoudji, M., Oocyte-specific inactivation of Omcg1 leads to DNA damage and c-Abl/TAp63-dependent oocyte death associated with dramatic remodeling of ovarian somatic cells. Cell death and differentiation 2015, 22, (1), 108-17.

16. Gonfloni, S.; Di Tella, L.; Caldarola, S.; Cannata, S. M.; Klinger, F. G.; Di Bartolomeo, C.; Mattei, M.; Candi, E.; De Felici, M.; Melino, G.; Cesareni, G., Inhibition of the c-Abl-TAp63 pathway protects mouse oocytes from chemotherapy-induced death. Nature medicine 2009, 15, (10), 1179-85.

17. Kim, S. Y.; Cordeiro, M. H.; Serna, V. A.; Ebbert, K.; Butler, L. M.; Sinha, S.; Mills, A. A.; Woodruff, T. K.; Kurita, T., Rescue of platinum-damaged oocytes from programmed cell death through inactivation of the p53 family signalling network. Cell death and differentiation 2013.

18. Reddy, E. P.; Aggarwal, A. K., The ins and outs of bcr-abl inhibition. Genes $\mathcal{E}$ cancer 2012, 3, (5-6), 44754.

19. Wylie, A. A.; Schoepfer, J.; Jahnke, W.; Cowan-Jacob, S. W.; Loo, A.; Furet, P.; Marzinzik, A. L.; Pelle, X.; Donovan, J.; Zhu, W.; Buonamici, S.; Hassan, A. Q.; Lombardo, F.; Iyer, V.; Palmer, M.; Berellini, G.; Dodd, S.; Thohan, S.; Bitter, H.; Branford, S.; Ross, D. M.; Hughes, T. P.; Petruzzelli, L.; Vanasse, K. G.; Warmuth, M.; Hofmann, F.; Keen, N. J.; Sellers, W. R., The allosteric inhibitor ABL001 enables dual targeting of BCR-ABL1. Nature 2017, 543, (7647), 733-737. Talpaz, M.; Hochhaus, A.; le Coutre, P.; Ottmann, O.; Heinrich, M. C.; Steegmann, J. L.; Deininger, M. W. N.; Janssen, J.; Mahon, F. X.; Minami, Y.; Yeung, D.; Ross, D. M.; Tallman, M. S.; Park, J. H.; Druker, 